\title{
Impact of the Interaction of Synchronous Machines and Virtual Inertia Provisions on the Small Signal Stability of Microgrids
}

Lalitha Subramanian $^{1^{*}}$, Mamadou Goundiam $^{2}$, Vincent Debusschere ${ }^{3}$, Hoay Beng Gooi ${ }^{4}$, Raphael Caire $^{5}$, Nouredine Hadjsaid ${ }^{6}$

\author{
${ }^{1}$ Energy Research Institute at NTU, Nanyang Technological University, Singapore \\ ${ }^{1-3,5-6}$ Univ. Grenoble Alpes, CNRS, Grenoble INP*, G2Elab, 38000 Grenoble, France * Institute of Engineering \\ Univ. Grenoble Alpes, France \\ ${ }^{4}$ School of Electrical and Electronics Engineering, Nanyang Technological University, Singapore \\ *Lalitha002@e.ntu.edu.sg
}

Keywords: DAMPING, FREQUENCY CONTROL, MICROGRID, SMALL SIGNAL STABILITY, VIRTUAL INERTIA

\begin{abstract}
\section{Introduction}

With the growing potential for local self-consumption using distributed energy resources (DERs), the concept of independently operable microgrids are becoming practical. Majority of DERs are power electronics-controlled, which lack synchronous inertia (SI) property, which is a necessary characteristic for the system resilience.

Consider an generation outage that causes the system frequency to drop, the initial rate-of-change-of-frequency (ROCOF) is determined by the SI of the system. SI resists the frequency change until the controllers respond and therefore allows the liberty of control delay in the primary frequency response (PFR). The higher the SI, the lesser the ROCOF and the lowest frequency dip $f_{\text {nadir }}$. Therefore, in low-inertia power systems, the inverters are controlled to be responsive to the system frequency and thereby synthetically emulate inertia.
\end{abstract}

With the increasing number of inverter-based resources in the distribution system, there is a growing possibility for the development of self-consuming energy communities. In the context of this transition, this work aims to study the interaction of synchronous and virtual inertia in low inertia microgrids, where the inertia is emulated synthetically by controlling the inverterbased resources to respond to the system frequency. The effect of the interaction of the grid-forming and grid-following inverter controls with synchronous machines on the oscillatory stability of the system has been explored.

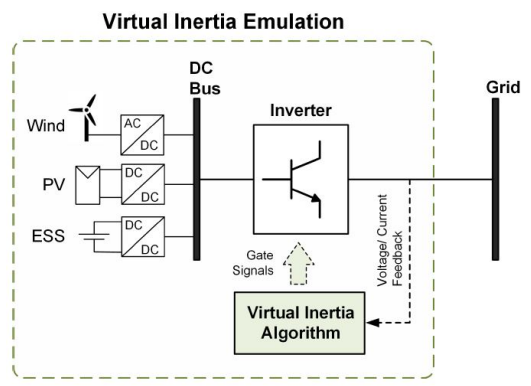

Fig. 1: Virtual Inertia Emulator [1]
Virtual Inertia Emulators (VIEs) are plug-and-play control devices that are connected to the inverter between the DC bus or energy storage or DER and the AC network as shown in figure 1. Inertia emulation is primarily associated with the enhancement of frequency stability. However, in this work, we intend to explore the small signal stability of low inertia systems with control of inverter-based resources (IBRs). It has been shown that the interaction of inertia controllers may enhance the frequency stability but deteriorate the small signal stability (SSS) of the system causing power oscillations [2, 3].

\subsection{State-of-art}

The control strategies proposed for the VIE range all the way from simple droop control to mimicking of synchronous generators. All the VIE devices follow the steps below to achieve the inertia response.

1. Measure the $\mathrm{AC}$ voltage/current/power/frequency.

2. Calculate the converter set points or references based on the droop logic or machine model.

3. Actuate the response by switching the actuator accordingly.

The main challenge associated with the inertia control is that the requirement for an extremely fast response after the occurrence of a disturbance. However, there will be delays in measurement, calculation, and actuation which makes it challenging. The individual VIEs react momentarily to the disturbances for maintaining the system equilibrium. The interactive oscillatory modes of VIEs must be sufficiently damped to ensure the oscillatory stability of the system. The control 
method, sizing, and position of the VIE greatly influence the oscillatory stability of the system. In the following section, the different types of inertia provision in the power system are modelled.

The small signal model of an islanded microgrid with $100 \%$ droop based IBR has been modelled in [4-6] and analyzed using modal analysis. Small signal stability of microgrids has been studied in the literature to ensure the reliable operation of MGs as they are low-inertia systems [7, 8]. The droop parameter is optimized with stability constraints in [5], however the requirement in this case is the parametric condition as it corresponds to a very fast control loop. Recently, conditions on the droop parameter has been derived for the lossless microgrid assumption using a Port-Hamiltonian representation [9]. In [10], a barrier function approach is taken to provide the safety conditions of the parameters in the microgrid. Recently, the small signal model of DERs with virtual inertia control has been derived in [11]. In this work, we intend to model the virtual inertia contribution of grid-forming and grid-following DERs with that of the synchronous machine model to analyze the impact.

\section{Small Signal Modeling}

In this sections, the inverter-based resources (IBRs) are modelled for small signal stability (SSS) analysis. The small signal model of the different types of inertia provisions is derived.

\subsection{Synchronous Machine}

The change in the electrical state of the synchronous machine, described by its swing equation in (1), affects the electromechanical oscillations, where $P_{m}$ is the mechanical power, $\omega_{m}$ is the turbine angular velocity, and $P_{e}$ is the electrical power. The electric power is a function of the rotor angle $\delta_{m}$ and the damping of electro-mechanical oscillations, which is why the transients induce currents in the rotor circuits.

$$
\begin{aligned}
\omega_{m} J_{m} \frac{d^{2} \delta_{m}}{d t^{2}} & =P_{m}-P_{e} \\
M=2 H & =\omega_{m}^{2} J / S \\
\frac{M}{\omega_{0}} \frac{d^{2} \delta_{m}}{d t^{2}} & =P_{m}-P_{e}-K_{d} \dot{\delta} \\
\Delta \omega_{r} & =\frac{\dot{\delta}}{\omega_{0}} \\
\dot{\Delta \omega_{r}} & =\frac{1}{M}\left(P_{m}-P_{e}-K_{D} \Delta \omega_{r}\right) \\
x & =\left[\begin{array}{c}
\delta \\
\Delta \omega_{r}
\end{array}\right]
\end{aligned}
$$

$M$ in (2) is the mechanical starting time. It is defined as the time taken by the machine to decelerate from synchronous speed to stand still when the mechanical power input is removed and rated electric power is extracted from the machine.

The inertia constant $H$ is calculated as twice the ratio of stored kinetic energy in the machine to the MVA rating $S$. If
$K_{d}$ is the damping coefficient (torque per unit speed deviation) provided by the damper windings in the rotor circuit and $\omega_{0}$ is the synchronous angular velocity, the swing equation becomes (3), where $\omega_{r}$ is the relative angular velocity in per unit. The state variables of the generator are given in (6).

Although the swing equation is the fundamental equation of power system dynamics and can be supplemented by algebraic equations, it does not account for the electro-magnetic dynamics, i.e. the dynamics of the rotor circuits and controls on field voltage. The disturbances cause changes in the electrical quantities of the rotor circuit, while the transients in the stator windings decay rapidly and can be neglected. The rotor circuit flux variations originate in the armature reaction effect of the stator field on the rotor currents and are modeled as follows.

$$
\begin{aligned}
& \dot{\Psi}_{f d}=\frac{\omega_{0} R_{f d}}{X_{a d u}} E_{f d}-\omega_{0} R_{f d} i_{f d} \\
& \dot{\Psi}_{1 d}=-\omega_{0} R_{1 d} i_{1 d} \\
& \dot{\Psi}_{1 q}=-\omega_{0} R_{1 q} i_{1 q} \\
& \dot{\Psi}_{2 q}=-\omega_{0} R_{2 q} i_{2 q} \\
& x=\left[\begin{array}{llllll}
\delta & \Delta \omega_{r} & \Psi_{f d} & \Psi_{1 d} & \Psi_{1 q} & \Psi_{2 q}
\end{array}\right]^{T}
\end{aligned}
$$

$f d, 1 d, 1 q$, and $2 q$ denote the field axis, d-axis damping circuit, and the q-axis damping circuits respectively. $\Psi$ represents the flux linkage; $i$ is the circuit current; $R$ is the resistance; $E_{f d}$ is the exciter output voltage; and $X_{a d u}$ is the unsaturated mutual impedance.

\subsection{Modeling Inverter-based Resources}

As part of the microgrid model, the IBRs with their controls and filters, lines, and loads are modelled in a linear state space representation.

2.2.1 Grid-forming Resources: Grid-forming IBR control is a voltage-source controlled strategy that allows the IBR to serve as a voltage source especially during the autonomous operation mode of the microgrid. The droop concept is most popular in power systems with several frequency responding units as it is a decentralized control with its natural synchronizing property.

The droop-controlled grid-formers act as voltage sources for the microgrid that respond to load changes by linear regulation of the output voltage and frequency, thereby facilitating power sharing amongst in a decentralized manner depicted by the droop law in (12), where $m_{p}$ and $n_{q}$ are the droop coefficients. This droop law is based on the assumption that the system impedance is predominantly inductive.

$$
\Delta \omega=-m_{p} \Delta P ; \Delta V=-n_{q} \Delta Q
$$

A typical inverter droop control scheme has been presented in figure $2 \mathrm{a}$. The droop block in figure $2 \mathrm{~b}$ measures the averaged values of real and reactive power given by (13) and (14) respectively. The active and reactive power measurements $P$ and $Q$ are computed from the inverter output current $i_{o}$ and voltage $v_{o}$. The measured voltages and currents are represented 


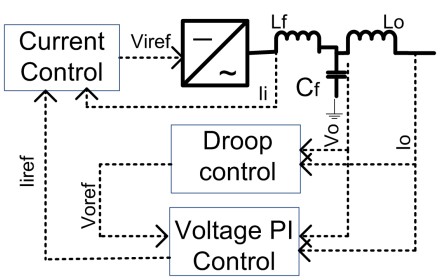

(a) Grid-forming Control

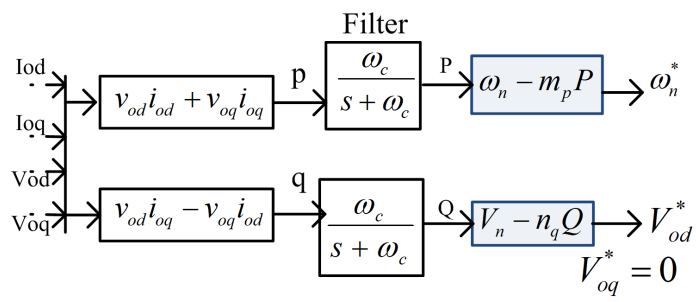

(b) Droop Control

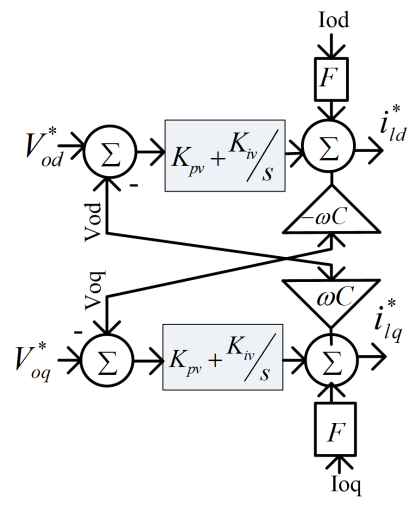

(c) Voltage Control

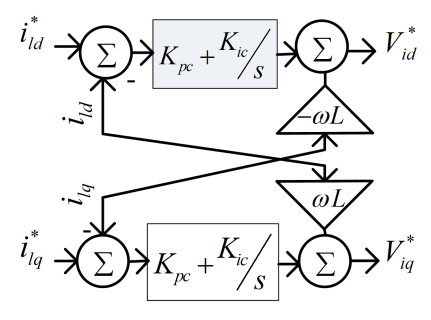

(d) Current Control
Fig. 2: Inverter droop control schematic.

in the $d-q$ reference frame as $\left(v_{o d}, v_{o q}\right)$ and $\left(i_{o d}, i_{o q}\right)$ respectively. In order to avoid the harmonics in the measurements from propagating to the control, a low pass filter (LPF) with corner frequency $\omega_{c}$ has been provided. The frequency and the voltage references are then generated based on the droop laws (15), where $\omega_{n}$ and $V_{n}$ represent the nominal frequency and voltage, $P_{\text {nom }}$ and $Q_{\text {nom }}$ are the nominal power outputs.

$$
\begin{aligned}
P & =\frac{\omega_{c}}{s+\omega_{c}}\left(v_{o d} i_{o d}+v_{o q} i_{o q}\right) \\
Q & =\frac{\omega_{c}}{s+\omega_{c}}\left(v_{o d} i_{o q}-v_{o q} i_{o d}\right) \\
\omega & =\omega_{n}-m_{p}\left(P-P_{n o m}\right) ; v_{o d}^{*}=V_{n}-n_{q}\left(Q-Q_{n o m}\right)
\end{aligned}
$$

The inverter is connected to the bus through an LCL filter with components $r_{f}, L_{f}, C_{f}, r_{c}$, and $L_{c}$. The voltage reference generated by the droop block, $\left(v_{o d}^{*}, v_{o q}^{*}\right)$ is fed to the inner voltage loop controller in figure $2 \mathrm{c}$ which computes the output current reference for the inverter given by (16)-(19). The corresponding PI control parameters are $K_{p v}$ and $K_{i v}$.

$$
\begin{aligned}
\phi_{d} & =\int\left(v_{o d}^{*}-v_{o d}\right) \\
\phi_{q} & =\int\left(v_{o q}^{*}-v_{o q}\right) \\
i_{l d}^{*} & =F i_{o d}-\omega_{n} C_{f} v_{o q}+K_{p v}\left(v_{o d}^{*}-v_{o d}\right)+K_{i v} \phi_{d} \\
i_{l q}^{*} & =F i_{o q}+\omega_{n} C_{f} v_{o d}+K_{p v}\left(v_{o q}^{*}-v_{o q}\right)+K_{i v} \phi_{q}
\end{aligned}
$$

The current reference $i_{l d}^{*}$ and $i_{l q}^{*}$ is fed to the current control loop in figure $2 \mathrm{~d}$ to generate the inverter output voltage reference before the LCL filter as given by (20)-(23). The corresponding PI control parameters are $K_{p c}$ and $K_{i c}$.

$$
\begin{aligned}
\nu_{d} & =\int\left(i_{l d}^{*}-i_{l d}\right) \\
\nu_{q} & =\int\left(i_{l q}^{*}-i_{l q}\right) \\
v_{i d}^{*} & =-\omega_{n} L_{f} i_{l q}+K_{p c}\left(i_{l d}^{*}-i_{l d}\right)+K_{i c} \nu_{d} \\
v_{i q}^{*} & =\omega_{n} L_{f} i_{l d}+K_{p c}\left(i_{l q}^{*}-i_{l q}\right)+K_{i c} \nu_{q}
\end{aligned}
$$

The inverter is connected to the bus through an LCL filter with components $r_{f}, L_{f}, C_{f}, r_{c}$, and $L_{c}$. The state space equations of the LCL filter are presented in (24)-(29).

$$
\begin{aligned}
\frac{d i_{l d}}{d t} & =-\frac{r_{f}}{L_{f}} i_{l d}+\omega i_{l q}+\frac{v_{i d}}{L_{f}}-\frac{v_{o d}}{L_{f}} \\
\frac{d i_{l q}}{d t} & =-\frac{r_{f}}{L_{f}} i_{l q}-\omega i_{l d}+\frac{v_{i q}}{L_{f}}-\frac{v_{o q}}{L_{f}} \\
\frac{d v_{o d}}{d t} & =\omega v_{o q}+\frac{i_{l d}}{C_{f}}-\frac{i_{o d}}{C_{f}} \\
\frac{d v_{o q}}{d t} & =-\omega v_{o d}+\frac{i_{l q}}{C_{f}}-\frac{i_{o q}}{C_{f}} \\
\frac{d i_{o d}}{d t} & =-\frac{r_{c}}{L_{c}} i_{o d}+\omega i_{o q}+\frac{v_{o d}}{L_{c}}-\frac{v_{b d}}{L_{c}} \\
\frac{d i_{o q}}{d t} & =-\frac{r_{c}}{L_{c}} i_{o q}-\omega i_{o d}+\frac{v_{o q}}{L_{c}}-\frac{v_{b q}}{L_{c}}
\end{aligned}
$$

The state space representation of the inverter has been written with respect to the inverter reference frame. Considering a microgrid with more than one inverter connected in parallel, the state space model has to be represented in the common reference frame by trasnformation matrices [6].

The overall state variables of each individual inverter may be listed as shown in (30). The additional subscript $i$ in (30) represents the state variables of a particular inverter $i$.

$$
\begin{array}{r}
\Delta x_{i n v}^{i}=\left[\begin{array}{rllllll}
\Delta \delta_{i} & \Delta P_{i} & \Delta Q_{i} & \Delta \phi_{d i} & \Delta \phi_{q i} & \Delta \nu_{d i} & \Delta \nu_{q i} \\
\Delta i_{l d i} & \Delta i_{l q i} & \Delta v_{\text {odi }} & \Delta v_{\text {oqi }} & \Delta i_{\text {odi }} & \Delta i_{\text {oqi }}
\end{array}\right]
\end{array}
$$

2.2.2 Grid-following Resources: Grid-following IBRs are essentially current-controlled distributed resources that rely on other sources or the grid to serve as the reference voltage source. The small-scale prosumer units in a microgrid may not be capable of performing regulation and stability functions. 
Grid-following PV inverters with power set point control are referred to as grid-feeding DERs. The grid-feeding units that inject a locally-set power to the grid based on their available resource and feed-in tariff. The grid-forming units act as reference voltage sources for these units. The power references $\left\{P_{r e f}, Q_{r e f}\right\}$ are used to compute the $d-q$ current references in (31). The computed reference currents are fed to the current control block.

$$
\left\{\begin{array}{l}
i_{l d}^{*}=\frac{2}{3}\left(\frac{P_{r e f} v_{d}-Q_{r e f} v_{q}}{v_{d}^{2}+v_{q}^{2}}\right) \\
i_{l q}^{*}=\frac{2}{3}\left(\frac{Q_{r e f} v_{d}-P_{r e f} v_{q}}{v_{d}^{2}+v_{q}^{2}}\right)
\end{array}\right.
$$
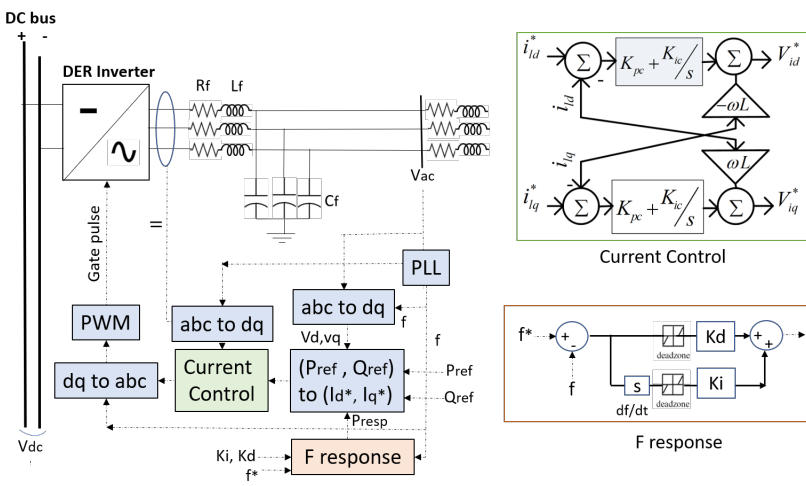

Fig. 3: Grid-following Control.

Consider the grid-feeding IBR in figure 3 , which is controlled as a current source to feed a reference power $P_{\text {ref }}, Q_{\text {ref }}$ set by the controller. Based on the power set-points, the reference current $I_{d, q}^{*}$ is calculated using the reference voltage from the grid-former or the main grid $v_{d}, v_{q}$. The state vector of a typical grid-feeding IBR is given by (34), where $\phi_{d c}$ is the state of the DC-DC PI control similar to $\nu_{i d}$ and $\nu_{i q}$ which are the states of the PI current controller, and $\theta_{p l l}$ belongs to the filter of the PLL. The input vector of the grid-feeding IBR consists of the $d-q$ components of the measured grid-former or main grid voltage as reference $v_{g}$, and the reference power inputs $P_{r e f}$ and $Q_{r e f}$ in (32). The outputs are the $d-q$ components of the IBR output current in the $D-Q$ reference $i o D$ in (33). The linearized state psace model of the grid-feeding IBR can therefore be computed based on the derived state space equations in Section ?? and the current controller equations similar to the droop control.

$$
\begin{gathered}
U_{\text {Gfeed }}^{i}=\left[\begin{array}{lllll}
v_{g D} & v_{g Q} & P_{\text {ref }, i} & Q_{\text {ref }, i}
\end{array}\right] \\
Y_{\text {Gfeed }}^{i}=\left[\begin{array}{lllll}
i_{o D i} & i_{o Q i}
\end{array}\right] \\
\Delta x_{\text {feed }}^{i}=\left[\begin{array}{llllll}
\Delta \delta_{i} & \Delta V_{d c-i} & \Delta \phi_{d c} & \Delta \theta_{p l l} & \Delta \nu_{d i} & \Delta \nu_{q i} \\
\Delta i_{l d i} & \Delta i_{l q i} & \Delta v_{o d i} & \Delta v_{o q i} & \Delta i_{\text {odi }} & \Delta i_{\text {oqi }}
\end{array}\right]
\end{gathered}
$$

Unlike grid-feeding IBRs, the grid-supporting ones respond to the frequency deviations by altering the power set point as a function of the system frequency. While the rest of the state space equations remain the same, the input vector also has the system frequency $\omega_{g}$ as a control variable. With the state space model of the inertia provisions, the microgrid model can be derived by coupling the machine, inverter, network and load models [6].

\section{Small Signal Analysis}

Figure 4 presents the eigenvalues of the system state matrix of the islanded IBR microgrid with grid-forming droop and inertia controllers and grid-feeding converters. The eigenmodes are divided into clusters based on the range of frequency $\omega$ and damping ratio $\zeta$.

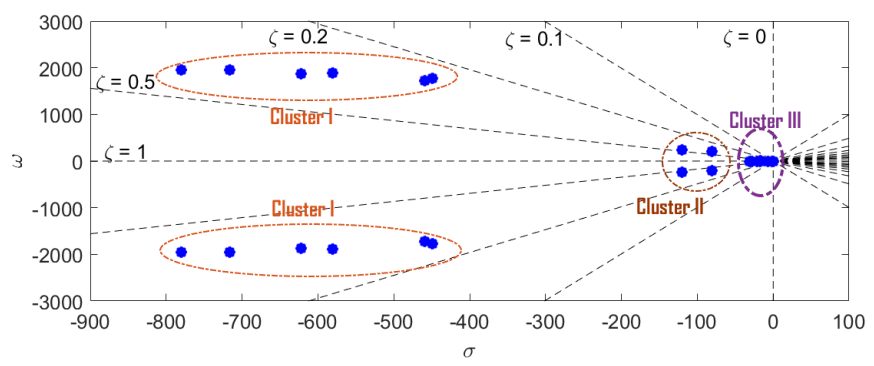

Fig. 4: Clustering the Eigenmodes of IBR Microgrid.

Cluster I comprises of high-frequency, low damping modes which correspond to the states dependant on the system impedances including line, load impedances and the LCL filter inductance and capacitance. Cluster I is related to the harmonic stability and power quality with high frequency oscillatory modes. Hence, the system operating conditions mostly affect these modes.

Cluster II has a high $\zeta$ and therefore are not oscillatory and do not impact the small signal stability. The modes of cluster II are sensitive to the states of the inner control loops such as current control in the grid-following IBR and the current and voltage controllers in the grid-forming IBR.

Cluster III is the most critical for the small signal stability assessment as it is the low-frequency mode with considerably low damping. The most critical mode is identified with a frequency of $20 \mathrm{~Hz}$, with $\zeta \approx 0.2$. The most critical modes correspond to the outer loop controls of IBRs, the droop controllers, and the PLL for the grid-following IBRs. Hence, the droop and damping parameters in grid-forming and grid-supporting control impact the small signal stability margin of the system.

\subsection{Impact of Parameter Variation on Eigen Traces}

In the sensitivity analysis with all droop-based IBRs, IBR 2 was the critical node, whose droop played a crucial role in determining the stability margin. To study the interaction between swing-based inertia control and droop controllers, the droop control of the IBR 2 has been replaced with the swing equation-based grid-forming inertia control. Thus, the control parameters for node 2 is $J$, while the other nodes are droopcontrolled. The modes of the inverter droop control (IDC) are referred to as inverter power modes and the modes of the 
swing equation-based control are referred to as the electromechanical modes, since the swing equation is based on a virtual rotor and prime-mover input power [4].

Increase in inertia $J$ shifts the inverter power modes towards the left-half of the s-plane and thereby stabilizing the system as shown in figure 5a. However, the increase in $J$, with droop constant $m_{p}$ as 0.05 , shifts the electro-mechanical modes of the swing-based controller to the right, making the stability margin narrower. Figure $5 \mathrm{~b}$ presents the effect of increase in $m_{p}$ when $J$ is constant at 0.03 . It can be observed that $m_{p}$ has the reverse effect of $J$ i.e. as the $m_{p}$ is increased the electromechanical modes are damped and the inverter power modes are destabilized. The critical $m_{p}$ at which the oscillatory modes become purely imaginary and the stability margin reduces to zero is computed as $16 \%$.

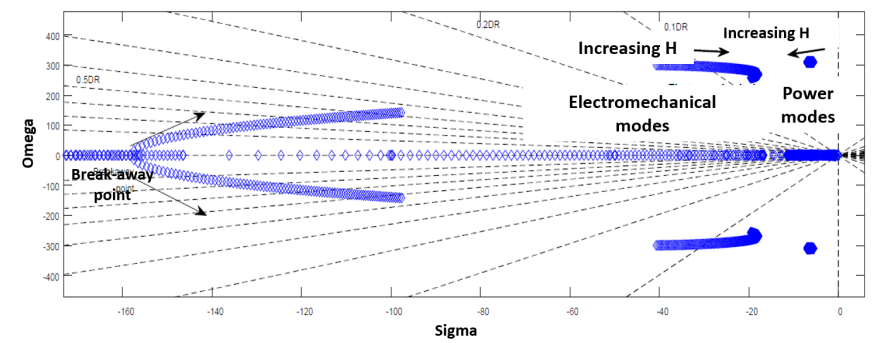

(a) Effect of increase of Inertia on Electromechanical and IDC Modes.

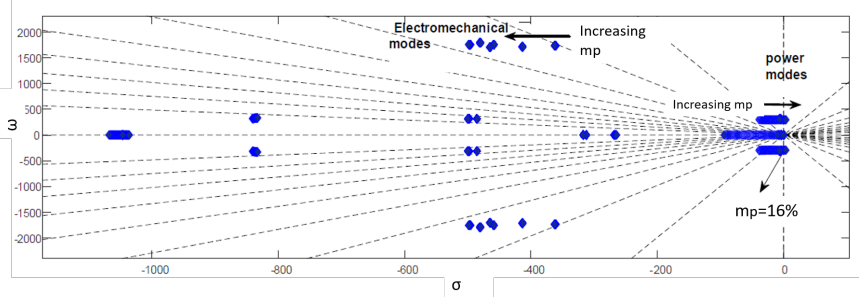

(b) Effect of increase of Droop on Electromechanical and IDC Modes.

Fig. 5: Interaction of Droop and Inertia Controllers.

From these plots, we can infer that if the $J / D$ ratio is increased the frequency damping increases while the active power damping reduces. Thus, it is possible to arrive at an optimal trade-off point [3] where both the frequency and the active power response are well-damped while minimizing the requirement of energy storage. For the droop control, the droop along with the corner frequency of the LPF $\omega_{c}$ determines the inertia and the damping is directly given by the droop. Thus, in case of the droop control, the $J / D$ has the constant value of $1 / \omega_{c}$ or $T_{f}$. If the $J / D$ ratio is less than $\omega_{c}$, the frequency response is over-damped and the power response is under-damped in case of droop control, and if the ratio is greater than $\omega_{c}$, the converse is true.

\section{Conclusion}

The impact of virtual inertia control provisions on the small signal stability of the system has been studied by modelling the different types of inertia provisions in the power system.
The small signal analysis has been carried out to analyze the effect of control parameter variations in grid-forming and gridfollowing controls. The interaction between the virtual and synchronous inertia provisions has been shown to result in additional inverter oscillatory modes, which will be analyzed in our future work.

\section{References}

[1] U. Tamrakar, D. Shrestha, M. Maharjan, B. P. Bhattarai, T. M. Hansen, and R. Tonkoski, "Virtual inertia: Current trends and future directions," Applied Sciences, vol. 7, no. 7, pp. 1-29, 2017.

[2] H. E. Brown and C. L. DeMarco, "Synthetic inertia and small signal instability," in 48th North American Power Symposium, 2016.

[3] P. Tielens and D. Van Hertem, "Influence of system wide implementation of virtual inertia on small-signal stability," in IEEE International Energy Conference, 2016.

[4] N. Soni, S. Doolla, and M. C. Chandorkar, "Inertia design methods for islanded microgrids having static and rotating energy sources," IEEE Industry Applications Society Annual Meeting, vol. 52, no. 6, pp. 1-8, 2015.

[5] E. Barklund, N. Pogaku, M. Prodanovic, C. HernandezAramburo, and T. C. Green, "Energy management in autonomous microgrid using stability-constrained droop control of inverters," IEEE Transactions on Power Electronics, vol. 23, no. 5, pp. 2346-2352, 2008.

[6] N. Pogaku, M. Prodanović, and T. G. Green, "Modeling, analysis and testing of autonomous microgrid of an inverter-based microgrid," IEEE Trans. on Power Electronics, vol. 22, no. 2, pp. 613-625, 2007.

[7] A. S. Vijay, D. K. Dheer, A. Tiwari, and S. Doolla, "Performance evaluation of homogeneous and heterogeneous droop-based systems in microgrid-stability and transient response perspective," IEEE Transactions on Energy Conversion, vol. 34, no. 1, pp. 36-46, 2019.

[8] D. K. Dheer, N. Soni, and S. Doolla, "Improvement of small signal stability margin and transient response in inverter-dominated microgrids," Sustainable Energy, Grids and Networks, vol. 5, pp. 135-147, 2016.

[9] J. Schiffer, R. Ortega, A. Astolfi, J. Raisch, and T. Sezi, "Conditions for stability of droop-controlled inverterbased microgrids," Automatica, vol. 50, no. 10, pp. 2457-2469, 2014.

[10] S. Kundu, W. Du, S. P. Nandanoori, F. Tuffner, and K. Schneider, "Identifying parameter space for robust stability in nonlinear networks: A microgrid application," Proceedings of the American Control Conference, vol. 2019-July, no. 1, pp. 3111-3116, 2019.

[11] S. Daarco and J. A. Suul, "Small-Signal analysis of an isolated power system controlled by a virtual synchronous machine," Proceedings - 2016 IEEE International Power Electronics and Motion Control Conference, PEMC 2016, pp. 462-469, 2016. 